\title{
CORRESPONDENCE
}

\section{Role of aspirin in patients with septic shock: a complex and intriguing relationship}

\author{
Marco Falcone ${ }^{1 *}$, Mario Venditti ${ }^{1}$ and Francesco Violi ${ }^{2}$
}

C 2016 Springer-Verlag Berlin Heidelberg and ESICM

\section{Dear Editor,}

Kiers et al. [1] raise an important issue related to the results of our recent report in which we demonstrated that patients with community-onset pneumonia on treatment with macrolides plus low-dose aspirin have improved survival compared to untreated ones [2].

Aspirin is a drug which irreversibly acetylates COX-1, so preventing formation of eicosanoids such as thromboxane $(\mathrm{Tx}) \mathrm{A} 2$, which aggregates platelets and prostaglandins. Improved survival rate could be dependent upon lowered formation of TxA2 and cardiovascular events, but Kiers et al. propose another more intriguing possibility. They argue, in fact, that as prostaglandins attenuate pro-inflammatory cytokine production at the transcriptional level aspirin, which inhibits protaglandin biosynthesis, they may counteract lung infection via an enhanced pro-inflammatory cytokine response to invading pathogens; this pro-inflammatory activity could result in more effective clearance of bacteria. The intriguing and provocative hypothesis of Kiers and colleagues may have another biologic plausibility, which has been demonstrated in a study performed in diabetic patients on chronic treatment with low-dose aspirin [3]. This study revealed that type II diabetic patients display an enhanced production of platelet F2-isoprostanes, which are chemically stable eicosanoids with pro-aggregating activity. Of note, F2-isoprostanes stem from arachidonic acid interaction with superoxide anion, which is a reactive oxidant species (ROS) formed by activation of Nox2, the enzyme of the innate immune system which is crucial for bacteria killing [3]. The authors speculated that, as a consequence of COX1 inhibition by aspirin, arachidonic acid is shifted towards Nox-2-derived ROS activation

\footnotetext{
*Correspondence: marco.falcone@uniroma1.it

1 Department of Public Health and Infectious Diseases, "Sapienza" University of Rome, Policlinico Umberto I, Viale dell'Università 37, 00161 Rome, Italy

Full author information is available at the end of the article
}

with ensuing enhanced production of isoprostanes [2]. Interestingly, an enhanced formation of isoprostanes has been detected in patients with coronary heart disease on chronic treatment with aspirin [4]. Based on this observation, it is possible that the improved survival by aspirin may be dependent on a paradoxical increase of inflammation, which is related not only to prostaglandin downregulation but also to a pro-oxidant activity derived from Nox2 activation. However, generalizability of this hypothesis needs further investigation.

\section{Author details \\ ${ }^{1}$ Department of Public Health and Infectious Diseases, "Sapienza" University of Rome, Policlinico Umberto I, Viale dell'Università 37, 00161 Rome, Italy. \\ ${ }^{2}$ Department of Internal Medicine and Medical Specialties, "Sapienza" Univer- sity of Rome, Policlinico Umberto I, Rome, Italy.}

Compliance with ethical standards

\section{Conflicts of interest}

The authors state that there are no conflicts of interest pertaining to this manuscript.

Accepted: 26 February 2016

Published online: 31 March 2016

\section{References}

1. Kiers HD, Kox M, van der Heijden WA, Riksen NP, Pickkers P (2016) Aspirin may improve outcome in sepsis by augmentation of the inflammatory response. Intensive Care Med. doi:10.1007/s00134-016-4264-0

2. Falcone M, Russo A, Farcomeni A et al (2016) Septic shock from community-onset pneumonia: is there a role for aspirin plus macrolides combination? Intensive Care Med 42:301-302. doi:10.1007/s00134-015-4139-9

3. Cangemi R, Pignatelli P, Carnevale R et al (2012) Platelet isoprostane overproduction in diabetic patients treated with aspirin. Diabetes 61(1626-32):16

4. Schwedhelm E, Bierend A, Mass R et al (2010) Redox-generated isoprostanes are associated with residual platelet activity in aspirintreated patients with stable coronary heart disease. J Thromb Haemost 8:2662-2670

\section{实

